\title{
A Discrete Model for Diffusion-induced Grain Boundary Deterioration
}

\author{
Andrey $\mathrm{P}$ Jivkov $^{\mathrm{a}}$ and John R Yates ${ }^{\mathrm{b}}$ \\ School of Mechanical, Aerospace and Civil Engineering, University of Manchester, UK. \\ aandrey.jivkov@manchester.ac.uk, bjohn.r.yates@manchester.ac.uk
}

Keywords: polycrystalline materials; grain boundary network; local diffusivity; discrete analysis; boundary cracking.

\begin{abstract}
Polycrystalline materials may suffer internal damage due to diffusion of chemically aggressive species during service. Diffusion rates are greatly enhanced on grain boundaries (GB). This can be modelled with discrete networks, where the GB structure is represented by links with local diffusivities. We present a site-bond model for concentration-driven diffusion that can be used to study the accumulation of chemical species at GB, leading to deterioration and eventual cracking. We employ realistic distributions of GB energies and corresponding diffusivities from published works. We show how the model can be used to predict macroscopic diffusivities with little experimentation. We demonstrate how the grain boundary structure controls the extent of internal damage resulting from the diffusion of chemical species.
\end{abstract}

\section{Introduction}

Grain boundaries (GB) have excess free volume and energy. This makes them fast diffusion paths, with diffusion rates several orders of magnitude greater than in the lattice [1]. The faster GB diffusion is responsible for changes at the length scale of the grain. Diffusion of constituent atoms leads to recrystallisation, grain growth, Cobel creep, dynamic embrittlement, and precipitation [2]. Others, such as oxidation and hydrogen embrittlement, result from diffusion of foreign species from the environment. The interest here is in foreign species diffusion, which can lead to cracking by several different mechanisms. Our starting point is that the diffusion of foreign species through polycrystalline materials can be represented by their diffusion along the GB network.

The temperature dependence of the diffusion coefficient is:

$$
D=D_{0} \exp (-Q / R T)
$$

where $D_{0}$ is a reference diffusivity and $Q$ is the activation energy. This is valid for lattice, surface or GB diffusion [3]; different diffusivities at a given temperature reflect differences in the activation energies. The enhanced diffusivity along a GB is due to reduced activation energy $Q_{G B}=Q_{L T}-\Gamma$, where $Q_{L T}$ is the lattice activation energy and $\Gamma$ is the GB excess energy, as a first approximation. Thus GB diffusivity, $D_{G B}$, is expressed via lattice diffusivity, $D_{L T}$, with

$$
D_{G B}=D_{L T} \exp (\Gamma / R T) \text {. }
$$

The link between GB structure and excess energy has long been considered and modelled through the coincident site lattice (CSL) formalism [4, 5]. However, it has become clear that the CSL classification cannot be mapped to energies. For example, the energies of $\Sigma 3$ boundaries alone can differ by an order of magnitude depending on the boundary plane. There are similar ranges for other boundary types [6]. One remedy is to consider a five-parameter description of the GB structure [7, 8]. This allows the mapping of energies to structures and determining GB areas as functions of energies. A recent study of ferritic steels reports an approximately linear relation between the logarithm of the GB area population and excess energies [9]. Such findings are very useful for modelling diffusion in GB networks, as they provide the probability of having a GB with given energy. Here we use the linear relation found in [9], which is reformulated as 


$$
\Gamma=\gamma-\sigma \ln (1-p)
$$

where $0 \leq \mathrm{p}<1$ is a uniformly distributed random number, $\gamma$ is the shift, and $\sigma$ is the shape of the excess energy distribution, in arbitrary units. Notably, $\sigma$ quantifies the network energy population; $\sigma$ $\approx 0.285$ is found in [9]. Larger values would describe networks with larger areas of higher energy boundaries, while smaller values - networks with reduced high energy boundaries, such as grain boundary engineered networks. Substitution of Eq. 3 into Eq. 2 leads to the expression

$$
D_{G B}=D_{P I}(1-p)^{(-\sigma / R T)} \text {. }
$$

where $D_{P I}=D_{L T} \exp (\gamma / R T)$ is a population independent diffusivity. Eq. 4 is used in this work to distribute diffusion coefficients to GB as a function of a random number $p$. For a fixed temperature, the ratio $\varepsilon=\sigma / R T$ measures a normalised network energy population. In this work we study the effect of $\varepsilon$ on the macroscopic steady-state diffusivity. We show how the results can be used with experimental data to calibrate $D_{P I}$. Finally, we illustrate how the model can be used to predict transient diffusion with associated degradation of grain boundaries.

\section{Model}

A polycrystalline material was modelled with space tessellation using truncated octahedra (unit cells), Fig. 1(a). The cell is the closest topologically to the average grain shape in real materials and offers some computational advantages for meso-scale modelling of solids $[10,11]$. The cellular structure was used to construct a discrete diffusion model, where sites were placed at the cell faces, Fig 1(b), and connected by conducting bonds between neighbouring faces, Fig. 1(c). The bonds were assigned diffusion coefficients according to Eq. 4. For a distribution of $p$ to bonds, models with $\varepsilon$ between 0.2 and 2.0 were analysed. These correspond to networks with increasing area of high energy GB. For comparison, if $\sigma=0.285$ is measured in $\mathrm{J} / \mathrm{m}^{2}$, and diffusion at $T=300 \mathrm{~K}$ is considered, $\varepsilon=1.64$; hence the studies covered both improved and degraded GB populations relative to the one reported in [9].
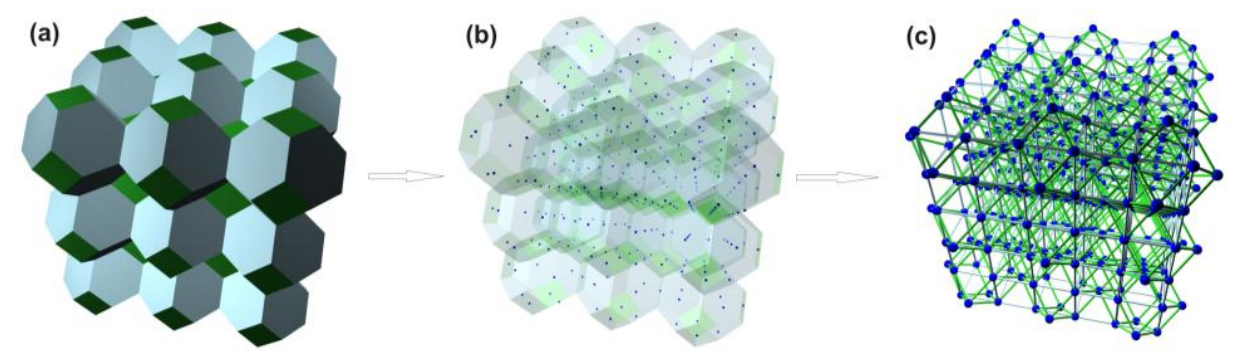

Figure 1. Discrete model for GB diffusion: (a) cellular representation of a polycrystal; (b) sites at cell faces; (c) site-bond model with bonds of variable diffusivities according to Eq. 4.

The models occupied the region $0 \leq X_{1} \leq 20 G ; 0 \leq X_{2} \leq 20 G ; 0 \leq X_{3} \leq 20 G$, where $G$ was the cell (average grain) size. The boundary conditions were: zero flux on planes $X_{2}=0, X_{2}=20 G, X_{3}=0$, $X_{3}=20 G$; and concentrations $C=C_{0}$ on $X_{1}=0$, and $C=0$ on $X_{1}=20 G$. These were used for calculating macroscopic diffusivity, $D_{m}$, under steady-state conditions using Fick's first law. Thus $D_{m}=F \times L / A$, where $F$ is reaction flux on $X_{1}=0$ or $X_{1}=20 G, L=20 G$ is diffusion length, and $A=$ $400 G^{2}$ is the crosssectional area. The models represent a reproducible experimental setup for measuring bulk diffusion coefficient of a material. In addition, transient solutions were performed with initial condition $C=0$ at all sites; concentration $C=C_{0}$ on $X_{1}=0$; and permeable film condition on $X_{1}=20 G$ to represent extension of the material in the $X_{1}$ direction with diffusivity $D_{m}$ found from respective steady-state solutions. It is assumed that a GB has deteriorated critically when $C \geq C_{f}$, where the critical concentration $C_{f}$ is related to the propensity of the GB to rupture upon load application. 


\section{Results and Discussion}

Five random assignments of $p$ to the bonds were used. For each assignment, steady-state analyses with bond diffusivities with varying $\varepsilon$ were performed. The results are shown in Fig. 2. It may be observed that the diffusivity predicted by the model, $D_{m}$, was found to be independent of the assignment of $p$, suggesting that the model size was sufficiently large to be representative of overall material. The logarithm of $D_{m}$, scaled with $D_{P I}$, was found to be a linear function of $\varepsilon$ with the parameters depicted. Notably, the constants of the linear fit, $a$ and $b$ are independent of the GB energy structure parameters, $\gamma$ and $\sigma$, and temperature. They arise from the topological structure of the GB network and the relationship given by Eq. (3). Therefore, if the cellular structure is a good representation of the polycrystal, and its GB population can be described by Eq. (3) with known slope $\sigma$, our finding allows the determination of $D_{P I}$ from experimental measurements of the macroscopic diffusivities of select species at two different temperatures. With calibrated $D_{P I}$, the physical diffusivities of the grain boundaries are known, Eq. (4), which allows for transient analysis of the diffusion.

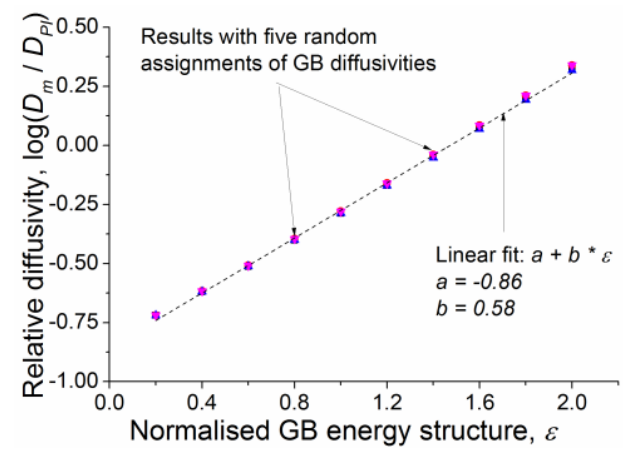

Figure 2. Dependence of macroscopic diffusivity on GB network energy population.

Transient analyses results are presented in two different ways. Firstly, the invasion of the foreign species is measured by the area of all GB with $C \geq C_{f}$; that is the GB susceptible to cracking. For illustration, the results for two selected $C_{f}$ are given in Fig.3. The evolutions follow dimensionless time; the physical time can be determined from the plots with calibrated $D_{P I}$ and specific grain size, $G$. The plots show the effect of GB energy population, from larger fraction of low-energy boundaries, $\varepsilon=0.8$, through to highly energetic populations, $\varepsilon=2.0$. The results can be used to determine GB deterioration with time for known energy population structure. Alternatively, they can be used to estimate the delay in intergranular damage if the GB energy population were improved.
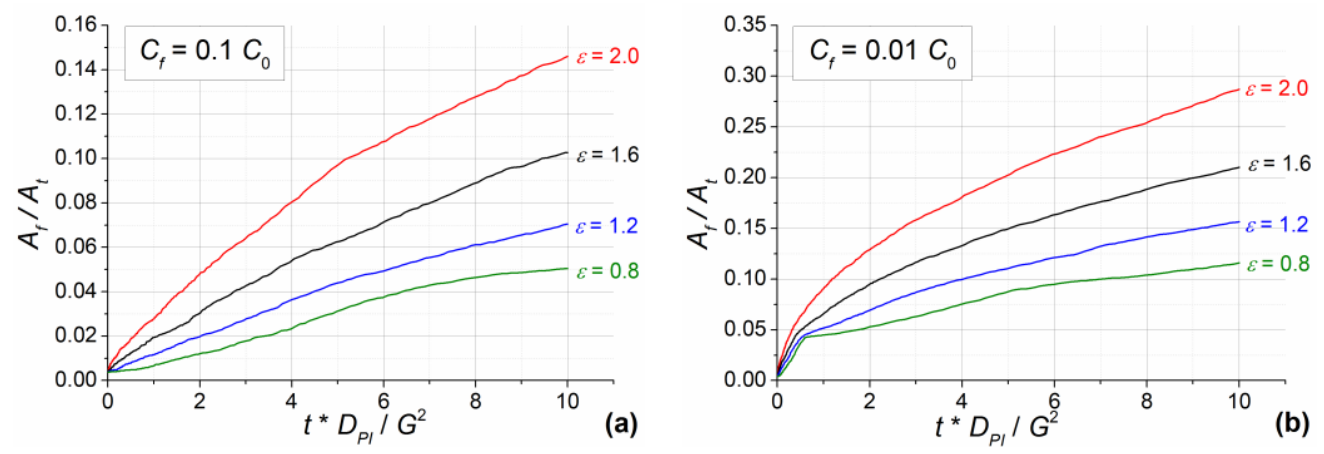

Figure 3. Evolution of crack-prone area for selected energy populations. Crackprone area, $A_{f}$ scaled with total GB area in the system, $A_{t}$.

Secondly, potential crack depths after fixed time of exposure were analysed. Results are given in Fig. 4 for the two selected $C_{f}$. The ordinates give the fractions of exposed GB from which cracks of corresponding depths could be expected. This can also be interpreted as surface area fractions; for $\varepsilon=2.0$ from Fig. 4(a) $5 \%$ of the surface is expected to be free from cracks; cracks of depths 0.25 $1.0 G$ are expected on $20 \%$ of the surface; and so on to the largest expected crack after the given time of exposure. The strong effect of the GB energy population is evident. Such analysis provides 
a powerful tool to assess the propensity for cracking of exposed surfaces and to estimate the maximum crack sizes after a particular time in service.
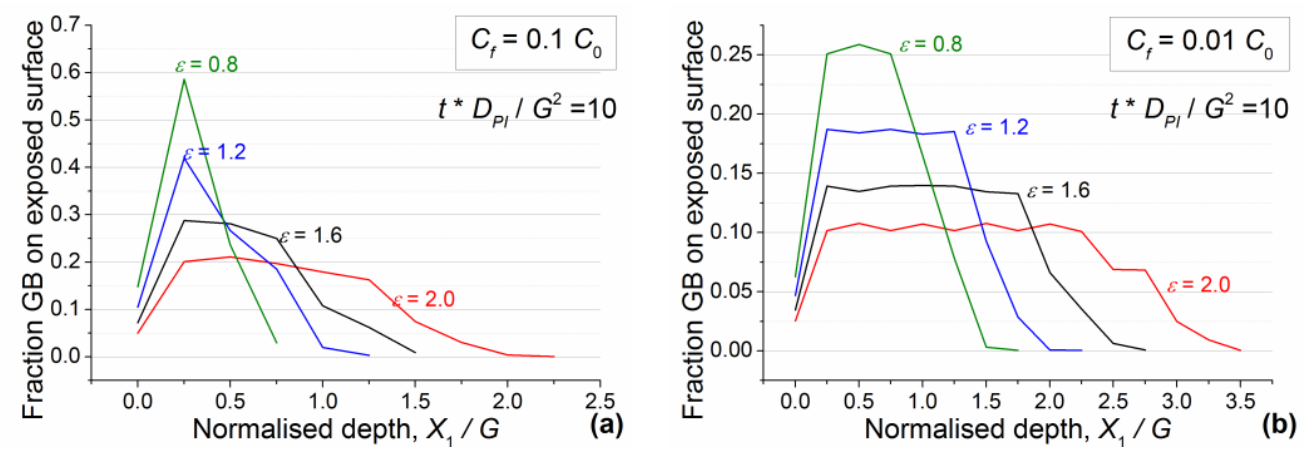

Figure 4. Potential crack depths from exposed surface for selected GB energy populations.

\section{Conclusions}

A discrete model for mass diffusion in polycrystalline materials was proposed. The scientific basis is enhanced diffusivity at GB due to their excess energies; and classification of GB networks by areas with different excess energies. This allows us to quantify the effect of GB energy populations on macroscopic diffusivity relative to a population-independent diffusivity. For a material with known energy population structure, the latter can be determined from experiments at two different temperatures. The results can then be used to assess the reduction in diffusivity with engineered improvements of the GB network. It was further shown that with calibrated population-independent diffusivity the model could be used to predict the evolution of intergranular damage with time, in terms of potential cracked area, as well as expected crack depths after a prescribed time.

\section{References}

[1] J.R. Fernandez, A.M. Monti and R.C. Pasianot: Metal. Mater. Trans. Vol. A33 (2002), p. 791.

[2] I. Kaur, Y. Mishin and W. Gust: Fundamentals of Grain and Interphase Boundary Diffusion (Wiley, Chichester, 1995).

[3] J.C. Fisher: J. Appl. Phys. Vol. 22 (1951), p. 74.

[4] B. Alexandreanu, B.H. Sencer, V. Thaveeprungsriporn and G.S. Was: Acta Mater. Vol. 51 (2003), p. 3831.

[5] Y. Chen and C.A. Schuh: Acta Mater. Vol. 54 (2006), p. 4709.

[6] D.L. Olmsted, S.M. Foiles and E.A. Holm: Acta Mater. Vol. 57 (2009), p. 3694.

[7] G.S. Rohrer: J. A. Ceram. Soc. Vol. 94 (2011), p. 633.

[8] S. Patala, J.K. Mason and C.H. Schuh: Prog. Mater. Sci. Vol. 57 (2012), p. 1383.

[9] H. Beladi and G.S. Rohrer: Acta Mater. Vol. 61 (2013), p. 1404.

[10] A.P. Jivkov, N.P.C. Stevens and T.J. Marrow: Comput. Mater. Sci. Vol. 38 (2006), p. 442.

[11] A.P. Jivkov and J.R. Yates: Int. J. Solids Struct. Vol. 49 (2012), p. 3089. 